\title{
Hybrid Supramolecular and Colloidal Hydrogels that Bridge Multiple Length Scales**
}

\author{
Emma-Rose Janeček, Jason R. McKee, Cindy S. Y. Tan, Antti Nykänen, Marjo Kettunen, \\ Janne Laine, Olli Ikkala,* and Oren A. Scherman*
}

\begin{abstract}
Hybrid nanocomposites were constructed based on colloidal nanofibrillar hydrogels with interpenetrating supramolecular hydrogels, displaying enhanced rheological yield strain and a synergistic improvement in storage modulus. The supramolecular hydrogel consists of naphthyl-functionalized hydroxyethyl cellulose and a cationic polystyrene derivative decorated with methylviologen moieties, physically crosslinked with cucurbit[8]uril macrocyclic hosts. Fast exchange kinetics within the supramolecular system are enabled by reversible cross-linking through the binding of the naphthyl and viologen guests. The colloidal hydrogel consists of nanofibrillated cellulose that combines a mechanically strong nanofiber skeleton with a lateral fibrillar diameter of a few nanometers. The two networks interact through hydroxyethyl cellulose adsorption to the nanofibrillated cellulose surfaces. This work shows methods to bridge the length scales of molecular and colloidal hybrid hydrogels, resulting in synergy between reinforcement and dynamics.
\end{abstract}

$\boldsymbol{R}$ solvent-based and solid nanocomposite architectures. ${ }^{[1-6]}$ Natural structural materials demonstrate a combined strength, stiffness, and toughness that are rarely achieved by synthetic materials, through bridging length scales and combining competing properties. Silk, animal bone, nacre, and plant fibers are all formed from self-assembled hierarchical structures across multiple length scales. Therein, hard reinforcing nanoscale domains are bound together by soft energy-dissipating molecular networks with sacrificial bonds, working together in synergy. Because of the highly complex structures, the synthetic production of related bioinspired networks and gels has proven to be challenging. However, some biomimetic materials exist, wherein "hard" colloidal reinforcement have been utilized to increase the strength and stiffness, in some cases even the toughness. ${ }^{[7-11]}$

One of the main strategies used by nature is the combination of colloidal-length-scale reinforcement with supramolecular bonds on a molecular level, thus allowing rapid dissociation and reformation. ${ }^{[12]}$ Thus prepared nanocomposites that incorporate molecular-scale networks with rapid supramolecular exchange kinetics in addition to colloidal reinforcement were explored herein. Engineering of sacrificial bonds into solid cellulose nanocrystal nanocomposites using supramolecular construction principles to achieve engineered fracture energy dissipation was recently demonstrated. ${ }^{[13]}$ Moreover, dynamic supramolecular bonds have been particularly attractive for self-healing supramolecular rubbers. ${ }^{[14]}$

The present study focuses on the combination of colloidal and molecular length scales in hydrogels to engineer a dynamic supramolecular sacrificial network that promotes stiffness and connectivity in nanofibrillated cellulose (NFC) colloidal hydrogels. Pure NFC forms hydrogels, in which the skeleton is formed by native cellulose nanofibers with lateral diameters of nanometers and lengths up to several micrometers. ${ }^{[15]}$ NFC has been used for a wide range of emerging applications, such as high-strength nanomaterials and biomedical scaffolds. ${ }^{[16-20]}$ NFC is extracted from plant cell walls and has high elastic moduli (26-36 GPa) and tensile strength in the GPa range. ${ }^{[21-23]}$ Dispersed NFC forms stiff hydrogels that are thought to be mediated by fibril-fibril entanglement facilitated by hydrogen bonding. ${ }^{[15]}$ However, the stiff crystalline domains of the nanofibrils allow little yielding and stress dissipation, hence the hydrogels prepared from NFC tend to be brittle. NFC hydrogels demonstrate shear thinning below $100 \%$ strain. Furthermore, there are challenges
[*] E.-R. Janeček, ${ }^{[+]}$C. S. Y. Tan, Prof. O. A. Scherman Melville Laboratory for Polymer Synthesis Department of Chemistry, University of Cambridge Lensfield Road, Cambridge CB21EW (UK) E-mail: oas23@cam.ac.uk

Dr. J. R. McKee, ${ }^{[+]}$Dr. A. Nykänen, Dr. M. Kettunen, Prof. O. Ikkala Molecular Materials, Department of Applied Physics Aalto University (previously Helsinki University of Technology) P.O. Box 15100, FIN-00076, Espoo (Finland)

E-mail: Olli.Ikkala@aalto.fi

Prof. J. Laine

Department of Forest Products Technology

School of Chemical Technology, Aalto University

P.O. Box 16300, FIN-00076 Aalto (Finland)

$\left.{ }^{+}\right]$These authors contributed equally to this work.
[***] This work was partially funded by the European Research Council Starting Investigator Grant (ASPiRe) ERC-2009-StG-240629, the Engineering and Physical Sciences Research Council grant EP/ F035535/1, the ERC Advanced Grant ERC 2011-ADG 291364 Mimefun, and the EPSRC. The Academy of Finland is acknowledged through the Center of Excellence of Molecular Engineering of Biosynthetic Hybrid Materials Research and an academy professorship (O.I.). J.R.M. acknowledges the ESF Precision Polymer Materials (P2M) network. O.I. and O.S. acknowledge the ESF Precision Polymer Materials (P2M) network for initiation of this work. The Aalto Nanomicroscopy Center is acknowledged for use of the devices. C.S.Y.T. acknowledges funding from the Department of Higher Education, Ministry of Education of Malaysia and the Universiti Teknologi MARA (UiTM) of Malaysia.

D Supporting information for this article is available on the WWW under http://dx.doi.org/10.1002/anie.201410570. 
a) Supramolecular hydroge

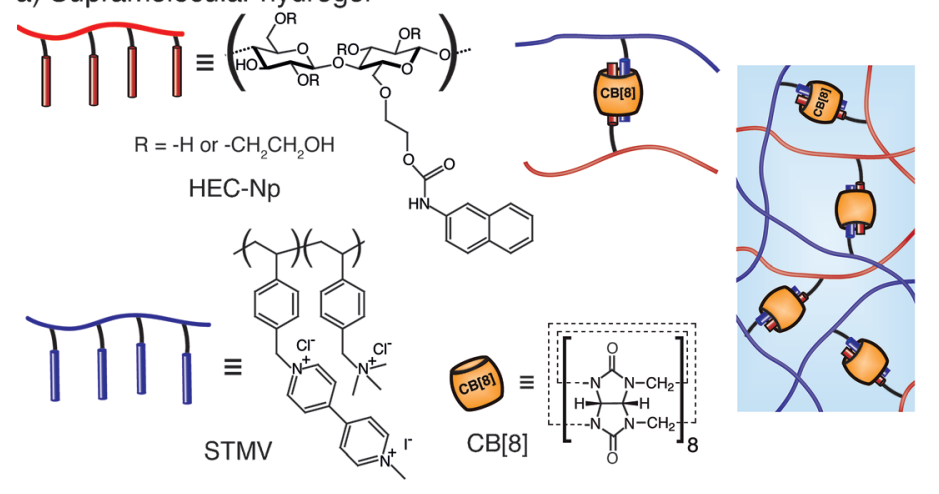

b) Colloidal reinforcing hydrogel

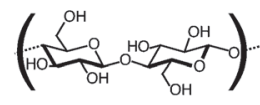

cellulose

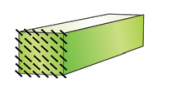

crystalline domain
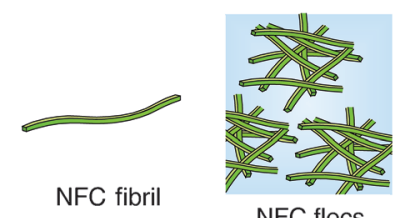

NFC flocs c) Hybrid hydrogel

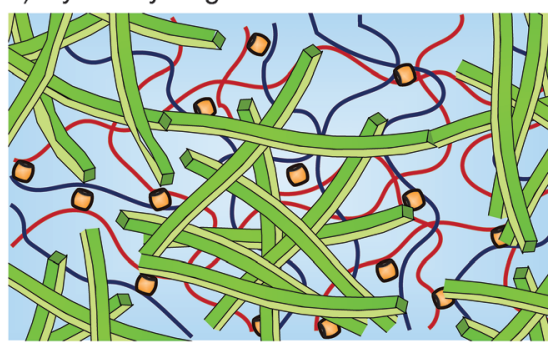

d) HEC-Np adsorption to NFC surface

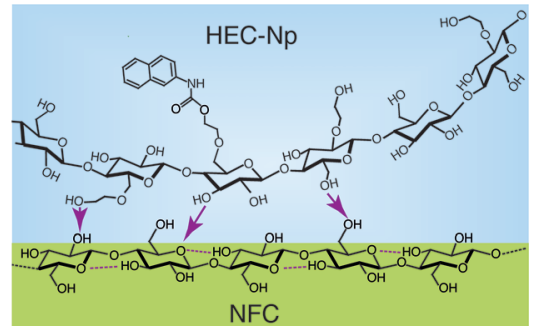

Figure 1. a) Supramolecular hydrogel consisting of HEC-Np, STMV, and the CB[8] host motif capable of binding the first guest naphthyl and the second guest viologen highly dynamically. b) Colloidal reinforcing nanofibrillated cellulose, also showing the denser and less dense network regimes (see Figure 5). c) Interpenetrating hybrid hydrogel consisting of the molecular-level supramolecular and colloidal-level NFC hydrogel. Because of its rigid nanofibers and short aspect ratio, network aggregates are formed (flocs). d) Surface adsorption of HEC-Np onto the NFC surface. Possible hydrogen bonding is schematically shown.

associated with achieving a homogeneous distribution in the aqueous medium. Within NFC hydrogels, denser "floc-like" regions typically form, separated by less dense "weak links" (Figure $1 \mathrm{~b}$, Figure $5 \mathrm{~b}$ ). ${ }^{[24,25]}$ A lack of dynamics within NFC cross-links coupled with separated material domains led us to the use of dynamic supramolecular linkages to enhance stiffness and yield strain, thus targeting significant challenges associated with the utilization of NFC hydrogels. NFC hydrogels are therefore our reference material herein.

Supramolecular interactions embedded within hydrogels have allowed for stimuli responsivity, controlled unzipping, and supramolecular cross-link reformations without immediate failure of the entire network. ${ }^{[26,27]}$ Specifically, the hostguest interactions of cucurbit[8]uril ( $\mathrm{CB}[8])$ display reversible complexation at rates approaching the diffusion limit. ${ }^{[28]}$ $\mathrm{CB}[8]$ can form 1:1:1 heteroternary complexes with two guest motifs, still allowing very high binding constants in spite of the dynamic nature. In this study, first-guest methylviologen (MV) and second-guest naphthyl (Np) functional polymers were used. Accordingly, the dynamic $\mathrm{CB}[8]$ network was formed using naphthyl-functionalized hydroxyethyl cellulose (HEC-Np) and a viologen functional styrene derivative (STMV; Figure 1a). Related polymeric constituents have previously been shown to be compatible with each other. ${ }^{[29]}$ Adsorbing functional polysaccharides, such as HEC, to a nanocellulose surface has also been demonstrated to be an effective method to form physically cross-linked nanocellulose networks. ${ }^{[30-32]}$ Favorable interfacial interactions improve the rheological properties of nanocomposite hydrogels. ${ }^{[29]}$ Hence, an interconnected system was foreseen whereby the HEC-Np "glued" the molecular-scale supramolecular network to the NFC colloidal scale network (Figure 1c).
In more detail, the architecture comprised of a colloidal NFC network enhanced by association with a dynamic supramolecular network (Figure 1c). A CB[8]-mediated supramolecular hydrogel, which displayed the favorable stiffness yet minimal solids content of fixed amounts of HEC-Np (5 mol\% Np loading), PSTMV (10 mol\% MV loading), and $\mathrm{CB}[8],{ }^{[33]}$ was investigated in combination with variable loading of the NFC hydrogel. A fixed composition of the supramolecular hydrogel was used, incorporating 0.5 wt. \% HEC-Np, 0.15 wt. \% STMV, and 0.1 wt. \% CB[8], which corresponds to nominal equimolar amounts of both naphthyl, viologen and $\mathrm{CB}[8]$. To this fixed composition, the NFC colloidal hydrogel was added (0-1.5 wt. \%). For reference, $\mathrm{CB}[8]$ was removed or replaced with $\mathrm{CB}[7]$ to show the essential role of the dynamic supramolecular interactions. Note that $\mathrm{CB}[7]$ does not allow simultaneous binding of viologens and naphthyls.

By combining a variable amount of NFC (0-1.5 wt. \%) with fixed amounts of STMV (0.15 wt.\%) and CB[8] (0.1 wt. \%), no apparent increase in the storage modulus was observed. Good compatibility was inferred as no apparent aggregation or phase separation occurred. Next, a fixed amount of HEC-Np (0.5 wt. \%) was added to form the heteroternary complex and to attach the ensuing supramolecular hydrogel to the NFC hydrogel through adsorbing the HEC to the NFC surface. This resulted in synergistic enhancement of the low-strain elastic modulus as the two networks were bound together (Figure 2a). The storage modulus data from the NFC colloidal hydrogel alone at varying weight content shows good agreement with a published model describing the rheological properties of NFC hydrogels. ${ }^{[34]}$ In order to carry out this fitting, a value of fiber Young's modulus of $31 \mathrm{GPa}$ was used based on published 
a)

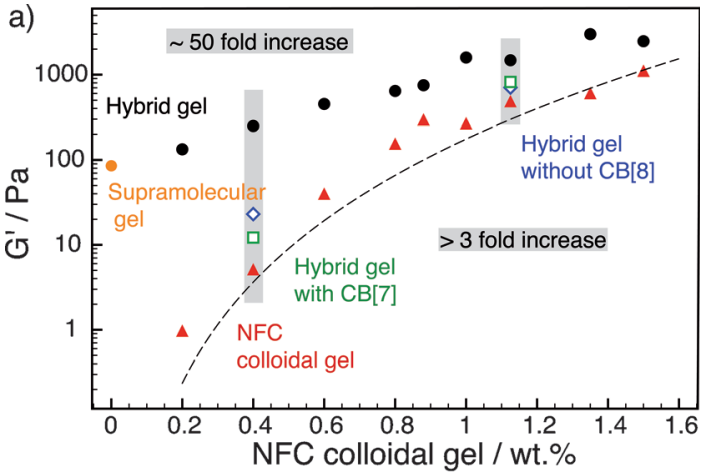

b)

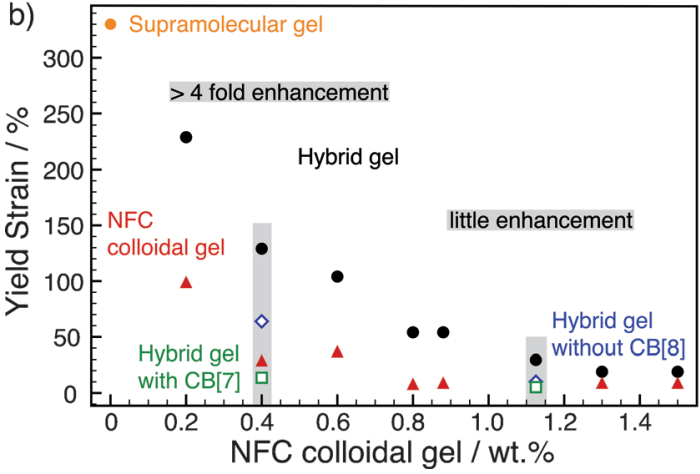

Figure 2. Rheology of the hybrid supramolecular and NFC colloidal hydrogel, as the concentration of the NFC colloidal gel is increased from 0 to $1.5 \mathrm{wt}$. \%, while the supramolecular hydrogel composition is kept fixed. a) Storage modulus determined in the linear regime $(0.1-$ $1 \%$ strain) at $10 \mathrm{rad} / \mathrm{s}$. The hybrid gel (black circles) has higher $\mathrm{C}^{\prime}$ values than either the corresponding NFC reference (red triangles) or the supramolecular gel (orange circle). Fitting of the pristine NFC data was done according to a published mode ${ }^{[34]}$ (dashed line). b) Yield strain determined at $10 \mathrm{rad} / \mathrm{s}$. For comparison, selected reference materials are also shown: hybrid hydrogel without $\mathrm{CB}[8]$ (blue diamonds), and hybrid hydrogel where $\mathrm{CB}[8]$ has been replaced by $\mathrm{CB}[7]$ (green squares).

data. ${ }^{[23]}$ A void volume fraction of 0.434 was calculated for the pure NFC colloidal hydrogel used here according to the fit shown in Figure 2. The stiffening of the hybrid gel was clearly mediated by the heteroternary $\mathrm{CB}[8]$ complex, as in its absence, rheological properties of the gel were only slightly increased relative to pristine NFC at the same concentration (Figure 2a, and Figure S1 in the Supporting Information), as shown by the low-strain modulus values. This result demonstrated that the specific $\mathrm{CB}[8]$-dependent interactions were essential for the promoted rheological properties. In the absence of $\mathrm{CB}[8]$, or using smaller host homologue $\mathrm{CB}[7]$, which does not form the ternary complex, only a slight increase in the storage modulus was observed.

As well as increasing the low-strain $G^{\prime}$, the addition of the $\mathrm{CB}[8]$ network to the NFC network resulted in increased yield strain (Figure 2b). It is normally observed that an increase in the covalent cross-linking density within a polymeric hydrogel will lead to an increase in $G^{\prime}$ and a decrease in yield strain. ${ }^{[35]}$ This is on account of the shorter distance between cross-links and hence a reduction in polymer elongation before pinning by a cross-link. Here, the addition of the dynamic supramolecular hydrogel bonding the NFC colloidal hydrogel domains together-resulted in an increase in $G^{\prime}$. Furthermore, $\mathrm{CB}[8]$ binding led to more dynamic interactions, allowing reconfiguration upon deformation, which resulted in enhanced elasticity and yield strain. However, this effect was less pronounced at high NFC weight content, at which the rheological properties of the hydrogel were dominated by the NFC.

In order to further investigate the hybrid hydrogel with different NFC colloidal gel loadings, but constant total solid content, the ratio between the two gel networks was varied while the total solid concentration was held constant at 1.15 wt. \%. As shown in Figure 3, there was no significant
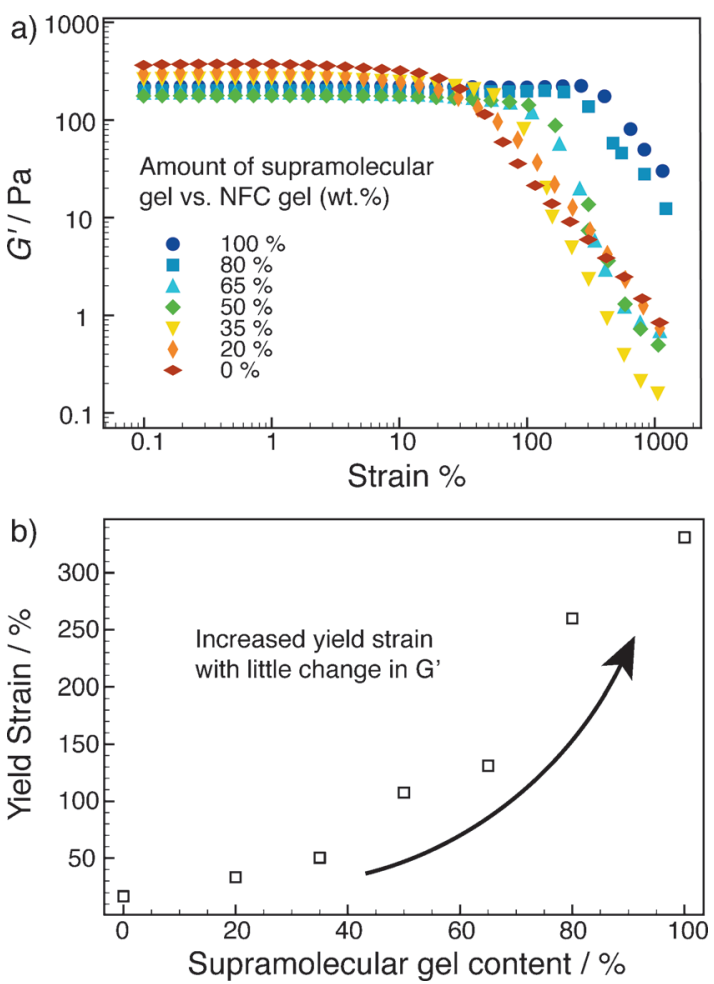

Figure 3. Rheology of the hybrid supramolecular and NFC colloidal hydrogel upon keeping the total amount of solids constant at 1.15 wt. \% with changing the weight content of the supramolecular gel vs. the NFC colloidal gel. a) Storage modulus. b) Yield strain.

increase in $G^{\prime}$ over the compositions investigated, however, the yield strain was increased by more than an order of magnitude, as the relative amount of the supramolecular hydrogel fraction increased. This observation can be explained by reconfigurations of supramolecular bonds, hence allowing the hybrid hydrogel to sustain greater shear strain before breakage of the overall nanocomposite. The change in yield strain with increasing supramolecular gel content falls between the curves calculated according to the rule of mixtures or the inverse rule of mixtures. This is as expected for a composite material with no alignment of fibers. Adherence to the rule of mixtures indicates interaction between the two hydrogel networks supporting the hypothesized mechanism of rheological enhancement. 

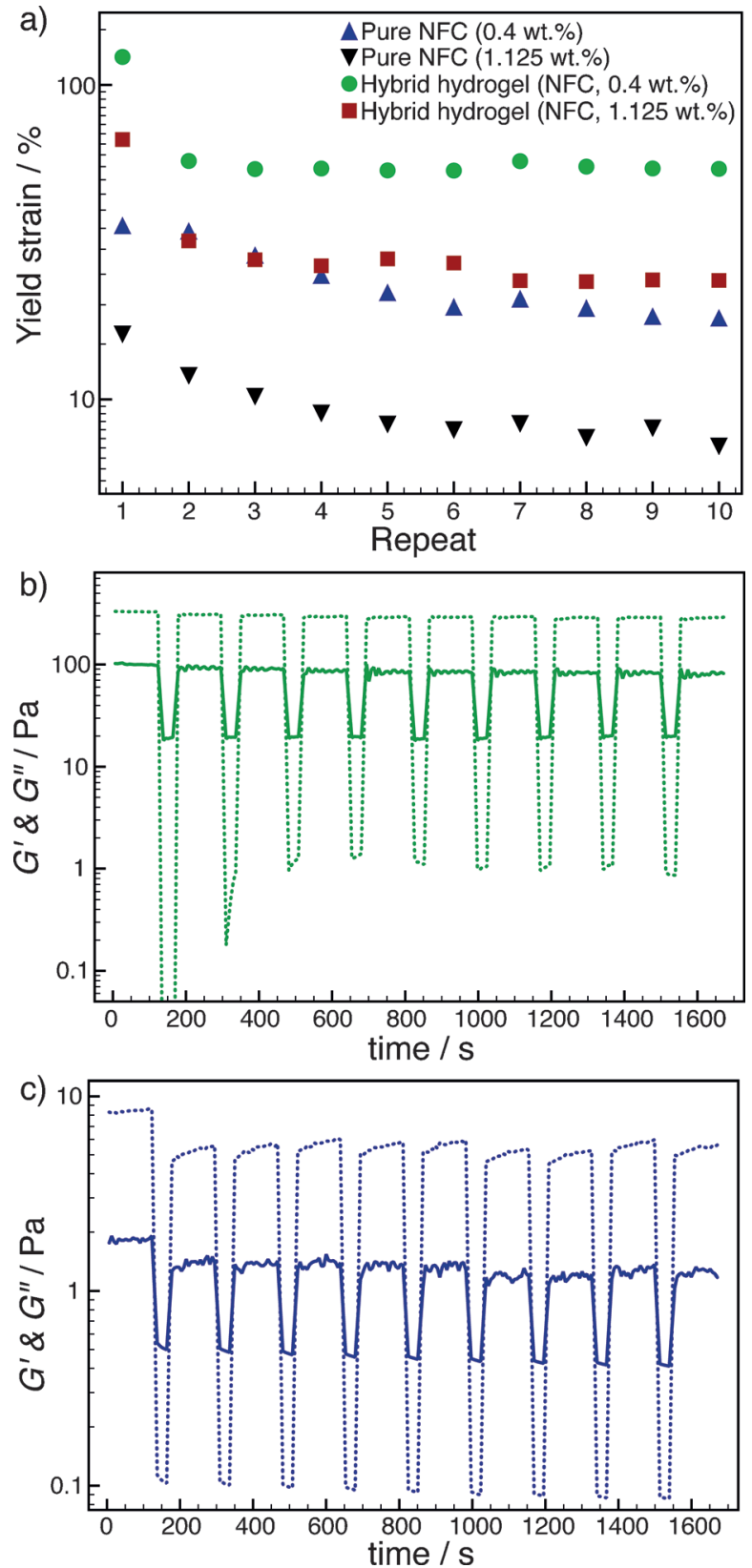

Figure 4. a) Sequential yield strain after breaking down the hydrogel network and allowing it to reform in repeated amplitude-sweep rheological experiments ( $\square$ ) hybrid hydrogel with $1.125 \mathrm{wt}$. \% NFC loading; ( $\mathbf{\nabla})$ reference pure NFC colloidal hydrogel 1.125 wt. \%; (•) hybrid hydrogel with $0.4 \mathrm{wt}$. \% NFC loading; ( $\mathbf{\Delta})$ reference pure NFC colloidal hydrogel $0.4 \mathrm{wt}$. \%. Yield strain calculated from the intercept of the mean $G^{\prime}$ over the first decade of strain \% with the $G^{\prime}$ slope after yielding. b, c) step-strain rheological experiments. b) Hybrid supramolecular and NFC colloidal hydrogels with $0.4 \mathrm{wt}$ \% NFC loading. c) Pure colloidal NFC hydrogel with $0.4 \mathrm{wt}$. \% loading. $G^{\prime}$ (dashed lines) and $G^{\prime \prime}$ (solid lines) measured over steps of $0.1 \%$ and $600 \%$ oscillatory strain.

Strain amplitude sweeps performed on pristine NFC colloidal hydrogels showed that once strained beyond the gelto-sol transition and retested, the low-strain $G^{\prime}$ was essentially unchanged (Figure S2); however, the yield strain was reduced with repeated gel-to-sol transitions (Figure $4 \mathrm{a}$ ). This obser-
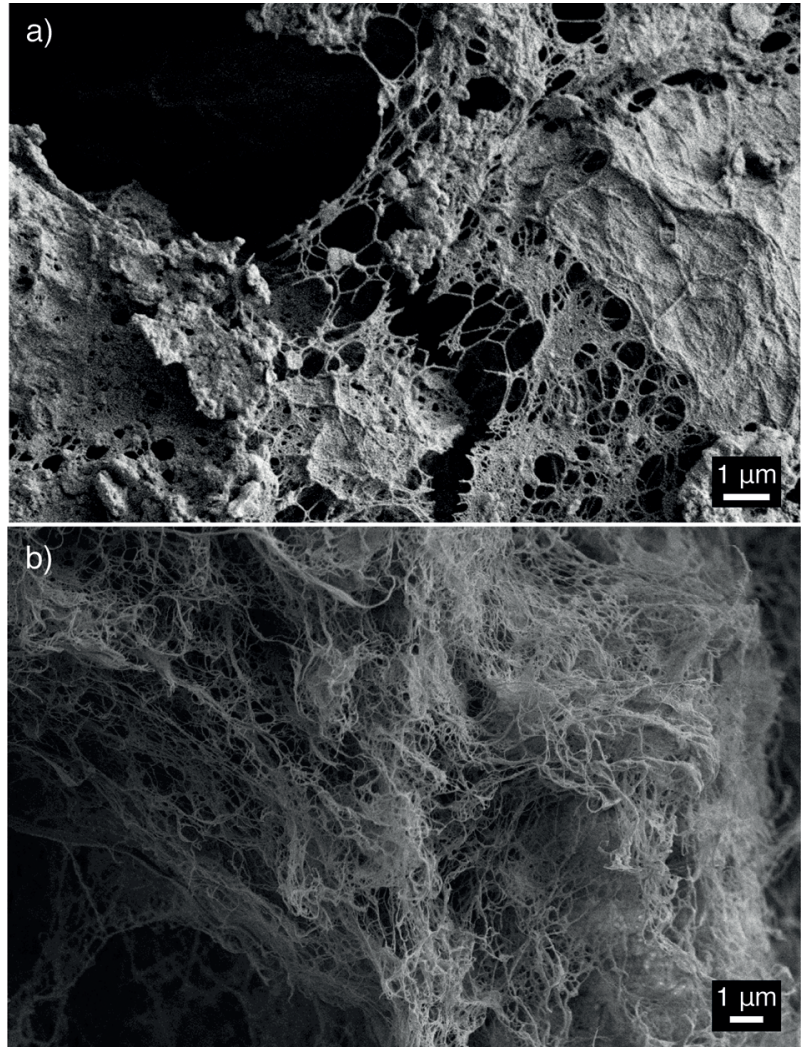

Figure 5. a) SEM image of the nanocomposite hydrogels with NFC loading (1 wt.\%). Note the supramolecular polymer between the fibrils. b) SEM image of the pristine NFC (1 wt.\%). Note the highly fibrillar structure.

vation suggests that the low strain $G^{\prime}$ was dependent on reversible entanglement and interactions between the NFC fibrils and hence was able to recover fairly quickly. However, the yield strain was likely dependent on the strongest entanglements within the NFC network, which were broken by each repeat of the sol-to-gel transition and hence the yield strain was decreased with each repeat (Figure $4 \mathrm{a}$ ). The hybrid supramolecular and NFC colloidal hydrogels, with the same supramolecular gel content as in Figure 2, however, demonstrated different behavior when repeatedly subjected to amplitude sweeps. Between repeat 1 and 2, there was a dramatically decreased yield strain, again indicating breakage of the strong, yet brittle NFC network. Yet, with subsequent repeats, the decrease in the yield strain was less pronounced. It is therefore proposed that not only was the hybrid hydrogel able to dissipate mechanical energy within the strained system, it was also able to mediate partial repair through rapid healing of the $\mathrm{CB}[8]$-based supramolecular gel network. The CB[8]-based supramolecular hydrogel alone is known to self-heal rapidly and completely upon shearing, hence this hydrogel cannot account for the decreased yield strain. $^{[33]}$

Step-strain experiments (Figure $4 \mathrm{~b}$ and c) illustrate this $\mathrm{CB}[8]$-mediated recovery. First, a $0.4 \mathrm{wt} \%$ pristine NFC colloidal hydrogel was subjected to $0.1 \%$ strain, well below the gel-to-sol transition, and then to $600 \%$ strain, well above the gel-to-sol transitions, and then retested at $0.1 \%$ strain. 
This cycle was then repeated to include ten low strain periods. It was found that although the initial $G^{\prime}$ was not fully recovered after the first cycle, a gel was again present at low strain, and additional cycles resulted in minimal decrease in the $G^{\prime}$. A significant time dependence of the $G^{\prime}$ was however noted, as during the $120 \mathrm{~s}$ at low strain, $G^{\prime}$ clearly increases. Note the curvature of the $G^{\prime}$ plot in all low strain periods after the first repeat (Figure $4 \mathrm{c}$ ). This suggests that re-entanglement of the NFC hydrogel was able to occur at room temperature under low-shear conditions. This experiment was repeated for hybrid hydrogels at higher NFC concentration and a similar trend was observed (Figure S3), however the $600 \%$ strain was no longer within the linear viscoelastic regime of the material. When the step-strain experiments were carried out on the hybrid supramolecular and colloidal hydrogels with 0.4 wt. \% NFC loading, an improved recovery in $G^{\prime}$ was achieved after the initial gel-to-sol transition and the time dependence on the $G^{\prime}$ recovery was less pronounced than for pristine NFC (Figure $4 \mathrm{c}$ ). This result suggests that the self-healing of the $\mathrm{CB}[8]$-based supramolecular gel network, thought to be in the order of the diffusion limit, was able to mediate recovery of the hybrid gel. Self-healing of the weak $\mathrm{CB}[8]$-based supramolecular gel network was therefore able to mediate healing of the NFC network, as the strength of the gel recovered was vastly above that of the $\mathrm{CB}[8]$-based supramolecular gel network alone. Rapid healing of the supramolecular hydrogel likely heals the interconnections of the dense regimes of NFC colloidal gels.

Finally, scanning electron microscopy (SEM) images support the schematic description of the network (Figure $1 \mathrm{c}$ and a), as the connections can clearly be seen between the NFC fibrils, whereas the pure NFC displays only a highly fibrillar structure (Figure 5b). Therefore, SEM illustrates the ability of the supramolecular hydrogel to bridge between the denser "floc-like" domains of the NFC colloidal hydrogel, leading to a reinforced hybrid nanocomposite. It is clearly illustrated, therefore the ability of the two hydrogel networks to interpenetrate and interact with each other. Cryo-TEM, on the other hand, demonstrates that the fibrillar structure remained intact even after the addition of the supramolecular network. This further indicated that all the components were compatible (Figure S4).

In conclusion, a supramolecular hydrogel bridges the colloidal nanofibrillar NFC hydrogel domains, leading to significantly enhanced storage modulus values and improved maximum elastic yield values. This was promoted by the fast dissociation/association dynamics of the supramolecular $\mathrm{CB}[8]$-based supramolecular cross-links in combination with the adsorption of hydroxyethyl cellulose component of the supramolecular hydrogel to the NFC nanofibers. The work shows biomimetic routes to combine components of different length scales and physical interactions to tune rheological properties.

Keywords: hydrogels · nanocellulose nanocomposites . supramolecular chemistry

How to cite: Angew. Chem. Int. Ed. 2015, 54, 5383-5388 Angew. Chem. 2015, 127, 5473-5478
[1] P. Fratzl, R. Weinkamer, Prog. Mater. Sci. 2007, 52, 1263.

[2] B. Bhushan, Philos. Trans. R. Soc. London Ser. A 2009, 367, 1445.

[3] H. D. Espinosa, J. E. Rim, F. Barthelat, M. J. Buehler, Prog. Mater. Sci. 2009, 54, 1059.

[4] G. E. Fantner, T. Hassenkam, J. H. Kindt, J. C. Weaver, H. Birkedal, L. Pechenik, J. A. Cutroni, G. A. G. Cidade, G. D. Stucky, D. E. Morse, P. K. Hansma, Nat. Mater. 2005, 4, 612.

[5] S. Keten, Z. Xu, B. Ihle, M. J. Buehler, Nat. Mater. 2010, 9, 359.

[6] M. A. Meyers, P.-Y. Chen, A. Y.-M. Lin, Y. Seki, Prog. Mater. Sci. 2008, 53, 1.

[7] Z. Tang, N. A. Kotov, S. Magonov, B. Ozturk, Nat. Mater. 2003, 2, 413.

[8] J. R. Capadona, O. van den Berg, L. A. Capadona, M. Schroeter, S. J. Rowan, D. J. Tyler, C. Weder, Nat. Nanotechnol. 2007, 2, 765.

[9] E. Munch, M. E. Launey, D. H. Alsem, E. Saiz, A. P. Tomsia, R. O. Ritchie, Science 2008, 322, 1516.

[10] A. Walther, I. Bjurhager, J. M. Malho, J. Pere, J. Ruokolainen, L. A. Berglund, O. Ikkala, Nano Lett. 2010, 10, 2742.

[11] L. J. Bonderer, A. R. Studart, L. J. Gauckler, Science 2008, 319, 1069.

[12] G. E. Fantner, E. Oroudjev, G. Schitter, L. S. Golde, P. Thurner, M. M. Finch, P. Turner, T. Gutsmann, D. E. Morse, H. Hansma, P. K. Hansma, Biophys. J. 2006, 90, 1411.

[13] J. R. McKee, J. Huokuna, L. Martikainen, M. Karesoja, A. Nykänen, E. Kontturi, H. Tenhu, J. Ruokolainen, O. Ikkala, Angew. Chem. Int. Ed. 2014, 53, 5049; Angew. Chem. 2014, 126, 5149.

[14] P. Cordier, F. Tournilhac, C. Soulié-Ziakovic, L. Leibler, Nature 2008, 451, 977.

[15] M. Pääkkö, M. Ankerfors, H. Kosonen, A. Nykänen, S. Ahola, M. Österberg, J. Ruokolainen, J. Laine, P. T. Larsson, O. Ikkala, T. Lindström, Biomacromolecules 2007, 8, 1934.

[16] S. J. Eichhorn, Soft Matter 2011, 7, 303.

[17] S. J. Eichhorn, A. Dufresne, M. Aranguren, N. E. Marcovich, J. R. Capadona, S. J. Rowan, C. Weder, W. Thielemans, M. Roman, S. Renneckar, W. Gindl, S. Veigel, J. Keckes, H. Yano, K. Abe, M. Nogi, A. N. Nakagaito, A. Mangalam, J. Simonsen, A. S. Benight, A. Bismarck, L. A. Berglund, T. Peijs, J. Mater. Sci. 2010, 45, 1.

[18] G. Siqueira, J. Bras, A. Dufresne, Polymer 2010, 2, 728.

[19] K. Shanmuganathan, J. R. Capadona, S. J. Rowan, C. Weder, Prog. Polym. Sci. 2010, 35, 212.

[20] K. Missoum, M. Belgacem, J. Bras, Materials 2013, 6, 1745.

[21] A. Šturcová, G. R. Davies, S. J. Eichhorn, Biomacromolecules 2005, 6, 1055 .

[22] T. Saito, R. Kuramae, J. Wohlert, L. A. Berglund, A. Isogai, Biomacromolecules 2013, 14, 248.

[23] S. Tanpichai, F. Quero, M. Nogi, H. Yano, R. J. Young, T. Lindstrom, W. W. Sampson, S. J. Eichhorn, Biomacromolecules 2012, 13, 1340.

[24] A. Karppinen, T. Saarinen, J. Salmela, A. Laukkanen, M. Nuopponen, J. Seppala, Cellulose 2012, 19, 1807.

[25] U. Bjorkman, Nord. Pulp Pap. Res. J. 2005, 20, 247.

[26] M. Guo, L. M. Pitet, H. M. Wyss, M. Vos, P. Y. W. Dankers, E. W. Meijer, J. Am. Chem. Soc. 2014, 136, 6969.

[27] J.-Y. Sun, X. Zhao, W. R. K. Illeperuma, O. Chaudhuri, K. H. Oh, D. J. Mooney, J. J. Vlassak, Z. Suo, Nature 2012, 489, 133.

[28] E. A. Appel, R. A. Forster, A. Koutsioubas, C. Toprakcioglu, O. A. Scherman, Angew. Chem. Int. Ed. 2014, 53, 10038; Angew. Chem. 2014, 126, 10202.

[29] J. R. McKee, E. A. Appel, J. Seitsonen, E. Kontturi, O. A. Scherman, O. Ikkala, Adv. Funct. Mater. 2014, 24, 2706.

[30] J. Laine, T. Lindström, G. G. Nordmark, G. Risinger, Nord. Pulp Pap. Res. J. 2000, 15, 520.

[31] I. Filpponen, E. Kontturi, S. Nummelin, H. Rosilo, E. Kolehmainen, O. Ikkala, J. Laine, Biomacromolecules 2012, 13, 736. 


\section{Angewandte}

Communications

[32] J. R. McKee, S. Hietala, J. Seitsonen, J. Laine, E. Kontturi, O. Ikkala, ACS Macro Lett. 2014, 3, 266.

[33] E. A. Appel, X. J. Loh, S. T. Jones, F. Biedermann, C. A. Dreiss, O. A. Scherman, J. Am. Chem. Soc. 2012, 134, 11767.

[34] R. J. Hill, Biomacromolecules 2008, 9, 2963.
[35] E. A. Appel, F. Biedermann, U. Rauwald, S. T. Jones, J. M. Zayed, O. A. Scherman, J. Am. Chem. Soc. 2010, 132, 14251.

Received: October 29, 2014

Revised: January 14, 2015

Published online: March 13, 2015 